\title{
Diseño e implementación de un plan de mantenimiento, basado en la metodología TPM, en planta productora de alimento balanceado para ganado bovino
}

\section{Design and implementation of a maintenance plan, based on the TPM methodology, in a balanced food production plant for bovine cattle}

\author{
HUMARÁN-SARMIENTO, Viridiana †*, PARRA-TELLEZ, Freddy Eduardo y CASTRO-LEAL, \\ Wilfrido
}

Instituto Tecnológico Superior de Guasave, División de Ingeniería Industrial

ID $1^{\text {er }}$ Autor: Viridiana, Humarán- Sarmiento /ORC ID: 0000-0003-0071-9181, CVU CONACYT ID: 240153

ID $1^{\text {er }}$ Coautor: Freddy Eduardo, Parra- Téllez /ORC ID: 0000-0002-1716-6301

ID $2^{\text {do }}$ Coautor: Wilfrido, Castro-Leal / ORC ID: 0000-0002-6434-8722

DOI: 10.35429/JIE.2019.9.3.26.35

Recibido 02 de Agosto, 2019, Aceptado, 14 de Septiembre, 2019

\begin{abstract}
Resumen
El caso de estudio presentado en esta investigación, aborda el análisis de la administración actual del mantenimiento de la maquinaria y equipo utilizada en la planta de mezclado de alimento balanceado para ganado bovino de una empresa privada de la región norte de Sinaloa, con la finalidad de diseñar e implementar una metodología eficiente para mejorar los paros no programados por las fallas recurrentes. Dentro de las actividades aplicadas, se realizó un ruteo de la maquinaria y equipos para diagnosticar el estado en que se encontraban, se aplicó la metodología 5's en el área de almacén y bodega para poder mantener el orden, limpieza y clasificación de herramientas, materiales y repuestos. Se diseñaron los formatos y bitácoras como un plan de mantenimiento integral, validándolo para efectuar los controles por varias semanas para comparar el diagnóstico inicial con los resultados finales. Se obtuvo un aumento de la productividad ya que disminuyeron los mantenimientos correctivos, disminuyendo tiempos muertos y desarrollando un compromiso con el personal manteniendo las áreas ordenadas, clasificadas y limpias.
\end{abstract}

Mantenimiento Productivo Total, Plan de Mantenimiento Preventivo, Mantenimiento Correctivo

\begin{abstract}
The case study presented in this investigation, addresses the analysis of the current management of the maintenance of the machinery and equipment used in the balanced feed mixing plant for cattle of a private company in the northern region of Sinaloa, with the purpose of design and implement an efficient methodology to improve unscheduled stoppages due to recurring failures. Within the applied activities, a routing of the machinery and equipment was carried out to diagnose the state in which they were, the 5's methodology was applied in the warehouse and cellar area to be able to maintain the order, cleaning and classification of tools, materials and spare parts. The formats and logs were designed as an integral maintenance plan, validating it to carry out the controls for several weeks to compare the initial diagnosis with the final results. An increase in productivity was obtained as corrective maintenance decreased, reducing downtime and developing a commitment with the staff, keeping the areas tidy, classified and clean.
\end{abstract}

Total Productive Maintenance, Preventive Maintenance Plan, Corrective Maintenance

Citación: HUMARÁN-SARMIENTO, Viridiana, PARRA-TELLEZ, Freddy Eduardo y CASTRO-LEAL, Wilfrido. Diseño e implementación de un plan de mantenimiento, basado en la metodología TPM, en planta productora de alimento balanceado para ganado bovino. Revista de Ingeniería Industrial. 2019 3-9: 26-35

\footnotetext{
* Correspondencia del Autor (Correo electrónico: viridianahumaranitsg@ gmail.com)

$\dagger$ Investigadora contribuyendo como primer autor.
} 


\section{Introducción}

Desde el principio de la humanidad hasta los siglos XVII y XVIII la prevención y mantenimiento que se le realizaban a las máquinas no tuvo un gran desarrollo debido a la menor importancia que tenían éstas con respecto a la mano de obra, por lo tanto el mantenimiento que se daba en esa época era de conservación correctiva. Sólo se arreglaba la máquina cuando presentaba paros o fallas y no se pensaba en el servicio que ésta suministraba. (Lopez, 2009) El Mantenimiento Productivo Total es una metodología de mejora que permite asegurar la disponibilidad y confiabilidad prevista de las operaciones, de los equipos, y del sistema, mediante la aplicación de los conceptos de: prevención, cero defectos, cero accidentes, y participación total de las personas (López, 2017).

En el presente artículo se presenta el desarrollo de una investigación aplicada en el periodo de junio del 2018 a junio del 2019. La cuál se realizó por una vinculación entre el Instituto Tecnológico Superior de Guasave y la empresa Ganadera Rubio's S.P R.L de C.V., como parte de un proyecto integral para la mejora de los procesos de mezclado y distribución de alimento para ganado bovino, una de las problemáticas que se detectaron fue la falta de un plan de mantenimiento, y paros no programados de la producción por averías en la maquinaria.

La empresa recurría a mantenimientos correctivos que eran lentos y a veces no se resolvían de manera inmediata lo que generaba disminución de la productividad diaria y afectación a la calidad y rendimiento del producto final, en este caso la engorda y salud del ganado.

Se designó al personal para el seguimiento del proyecto, y se programaron las actividades para el lograr el objetivo general de diseñar e implementar un plan de mantenimiento preventivo, con el enfoque metodológico de mantenimiento productivo total (TPM), el cual hace énfasis en el cambio de cultura organizacional, desde la dirección general de la empresa y todos los procesos involucrados para el aseguramiento de la calidad, contribuyendo con esta investigación la aplicación de metodologías de Ingeniería a la solución de problemáticas reales en el sector productivo.
Fue un proceso largo y difícil sobre todo por la actitud $\mathrm{y}$ hábitos de trabajo muy arraigados en la organización, sin embargo se avanzó mucho en el diagnóstico, diseño y seguimiento de bitácoras, planes y manuales propuestos y validados para retroalimentación de los controles establecidos, para la mejora de los mismos.

\section{Estado del arte}

La implementación de un plan de Mantenimiento Total de la producción es de gran ayuda, ya que con este se reducen costos de reparación de la maquinaria que se somete a dicho plan (Sacristán, 2018). Con este contexto, se mencionara una revisión de otros autores, que han llevado a cabo estudios similares.

Guachisaca Carlos y Salazar Martha en su tesis "Implementación de 5's como una metodología de mejora en una Empresa de elaboración de pinturas" en el 2009, encontraron en dicha empresa como principal problemática la acumulación de materiales innecesarios tanto como producto en proceso, desperdicios e insumos, desperdicio en la búsqueda de material de envase y de herramientas, largos tiempos de preparación, limpieza y poco trabajo en equipo. Para ello se implementaron la metodología 5's dentro de las dos áreas más críticas del proceso de elaboración de pinturas, con un estudio realizado con el fin de lograr un ambiente de trabajo productivo, seguro y confortable. Posteriormente se obtuvieron resultados muy favorables que por consiguiente realizaron un VSM propuesto en cada una de las áreas para medir y evaluar las mejoras logradas tanto en el área de producción como en bodega de material de empaque, mediante la comparación de indicadores de medición antes y después de la implementación de la metodología 5's. (Guachisaca \& Salazar, 2009).

Montoya Iván y Parra Carlos en su trabajo de grado "Implementación del Total Productive Management (TPM) como tecnología de gestión para el desarrollo de los procesos de maqui avícola" en Agosto de 2010, desarrollaron un plan de implementación de dicha metodología en una empresa del sector avícola colombiano conocida como MAQUIAVICOLA LTDA. 
Esta implementación tiene por objetivo optimizar la ejecución de los procesos productivos que se desarrollan dentro de la empresa con el fin de incrementar su productividad.

A partir de la descripción de los conceptos y herramientas que contempla esta filosofía, se diseña un plan de implementación que va a permitir aplicar este modelo al desarrollo de los procesos diarios de la empresa, contribuyendo a la mejora de indicadores de gestión. (Montoya \& Parra, 2010)

Mansilla Natalia en su informe "Aplicación de la metodología de mantenimiento productivo total (TPM) para la estandarización de procesos y reducción de pérdidas en la fabricación de goma de mascar en una industria nacional" en Marzo de 2011, realizó un estudio para implementar la metodología paso 5 TPM en Industria de Alimentos Arcor - Dos en Uno, donde se fundamentó en la inspección de los procesos con el propósito de lograr una estandarización y la disminución pérdidas en la fabricación de chicle en dos líneas de producción llamadas línea 1 chicle sin azúcar y línea 2 chicle con azúcar.

Luego de la implementación de paso 5 y la estandarización del proceso, se demostró que se logró reducir la cantidad de defectos de calidad como productos no conformes (línea 1: $57 \%$ y línea 2: $82 \%$ ); disminuir las paradas de equipos por fallos de proceso, tales como el número total de fallos de proceso (línea 1: $54 \%$ y línea 2: $2 \%$ ), número de fallos de proceso del departamento calidad (línea 1: 68 \% y línea 2: 45 $\%)$ y la variación de medida, principal causa de los fallos (línea 1: $13 \%$ y línea 2: $27 \%$ ). También se redujo los impactos ambientales mediante el uso racional de recursos: el scrap disminuyó un $27 \%$ en la línea 1 y un $13 \%$ en la línea 2; reproceso disminuyó un $48 \%$ en la línea 1 y un $100 \%$ en la línea 2. Además se evitaron los accidentes laborales. (Mansilla, 2011).

Marin Juan y Martínez Rafael en su artículo "Barreras y facilitadores de la implantación del TPM" en Julio de 2012, pretenden aproximarse a esa dificultad y aportar un análisis que permita avanzar en su conocimiento. Estos autores se dan cuenta de que las empresas modernas precisan ser competitivas para sobrevivir.
Esta necesidad es especialmente importante en momentos de crisis económica e incertidumbre, donde las empresas requieren de flexibilidad para garantizar su rentabilidad, a través de ajustes en su estructura organizacional y productiva. Para conseguir aumentar la competitividad, muchas compañías se plantean estrategias como el TPM para la obtención de mejoras tangibles e intangibles en la organización que les ayuden a afrontar las dificultades del negocio. Obteniendo como resultados de la implementación del TPM un impacto positivo que su desarrollo tiene en las organizaciones y su amplia utilización en grandes corporaciones, así como la dificultad que presenta la implantación de su modelo de desarrollo. $\mathrm{Su}$ aportación principal son las causas que originan esta dificultad y cómo éstas influyen en la consecución de los objetivos de cada paso del modelo de implantación. (Marín \& Martínez, 2012)

Mendoza José Luis en su trabajo de investigación de "Revisión sistemática en plan de mantenimiento preventivo", hace referencia al beneficio del hombre y su progreso, concerniente al desarrollo de estrategias de prevención de riesgos, implementando sistemas de prevención en la maquinaria utilizadas en distintas áreas de las empresas, de manera paralela a la mejora continua, para minimizar riesgos a través de un plan de mantenimiento preventivo actualizado y de acuerdo a las normas ISOS en calidad y medio ambiente. (Mendoza, 2018).

Rojas Enciso William, en su trabajo de investigación titulado "Mantenimiento autónomo para mejorar la calidad de servicio en el área de operaciones de la empresa J\& S ingenieros consultores E.I.R.L - 2018”, Utiliza herramientas como el diagrama de Ishikawa, midiendo cuantitativamente las razones asociadas a las 6M (mano de obra y métodos $31 \%$, y maquinaria, material y medio ambiente con un $13 \%$ cada uno). Además aplico la metodología del mantenimiento autónomo en la línea de conversión de rollos de la empresa papelera, reduciendo considerablemente los días perdidos en producción y las toneladas de producción del 2017 al 2018 respectivamente, además calculó el beneficio económico, de impacto para la empresa, con un tiempo de recuperación de 4 meses después de la implementación del mantenimiento autónomo. 
Marrero Hernández, José Alberto et al, en su artículo "Model diagnostic-maintenance planning and control" presenta un modelo con enfoque de cadena de valor, con los objetivos de 1. Compilar información sobre la planificación de la gestión del mantenimiento, 2. Integrar en un modelo gráfico las actividades de diagnóstico, planificación y control del mantenimiento como parte de la gestión, y 3. Garantizar la planificación del mantenimiento basado en técnicas multicriterio que permita la mejora de este proceso, integrando herramientas y métodos cuantitativos, para contribuir a la toma de decisiones. Dentro de los beneficios de este modelo se logró aumentar el rendimiento de la empresa y que las ventas crecen $95 \%$ más que los costos de operación; aumentando en un 56\% el plan de venta con respecto al método actual de planificación. (Marrero, 2019).

Es notable que la implementación del mantenimiento total de la producción ha generado gran impacto en las organizaciones en las empresas que se ha ejecutado, sin embargo como la filosofía de las herramientas "lean manufacturing", el éxito de las mismas depende del compromiso integral, y de la mejora continua.

\section{Metodología}

El método que se utilizó para el desarrollo del proyecto fue la metodología basaba en los pilares de TPM (Mantenimiento Productivo Total), acotada a las necesidades y políticas de la empresa, como las metodologías 5’S, Análisis de Modo y Efecto de Fallas (AMEF) y entrevistas directas con el personal encargado de mantenimiento.

Como instrumentos y técnicas de medición se utilizaron formatos de tipo cualitativo, y la observación directa en los procesos productivos, hojas de verificación y registros.

A continuación se detallan las
actividades realizadas.

Actividad 1: Análisis y recolección de datos: Se analizaron los equipos de las instalaciones de la planta a través de un ruteo de los equipos durante algunas semanas para recolectar datos a través de un diagnóstico de los equipos y así poder identificar problemas de los equipos.
Actividad 2: Implementación de la metodología 5's: Se implementó la metodología 5's en las áreas de taller para mantener ordenadas y clasificadas las herramientas de trabajo y realizando limpieza de toda el área para mantener una buena imagen; así como también se implementó en el área de almacén de materiales y repuestos para ordenarlos y clasificarlos.

Con esta metodología se ayudara al flujo de entradas y salidas de materiales y repuestos, así como la agilización en reparación de los equipos.

Actividad 3: Investigación del mantenimiento de los equipos: Se investigó a fondo los componentes, tipo de mantenimiento que se debe realizar y la frecuencia con la que se debe realizar. También se indago sobre quién debe realizar el mantenimiento, los costos de cada mantenimiento, herramientas que se utilizan a la hora de realizarlo y repuestos y materiales necesarios que se deben utilizar o de los cuales se debe manejar un stock mínimo.

Actividad 4: Diseño del plan de mantenimiento total de producción: Se elaboró un plan de mantenimiento preventivo anual de cada uno de los equipos; se enlistaron una serie de actividades a realizar semanal, mensual, trimestral, semestral y anualmente en el cual se especifica la frecuencia con la que se le debe realizar cada mantenimiento.

Actividad 5: Implementación del plan de mantenimiento: Después de conocer las necesidades de los equipos de la plata se realizaron mejoras al plan de mantenimiento como lo son incluyendo más actividades a realizar más específicas, seccionando los meses del año por semanas para facilitar la lectura del plan de mantenimiento y así mismo su llenado; también se implementó una bitácora semanal especificando las actividades que se deben realizar frecuentemente a corto plazo y así poder llevar un mejor control de los mantenimientos que se van realizando en el transcurso de la semana.

Se ejecutó implementación del plan durante 8 semanas. Además se realizó un Análisis de Modo y Efecto de Fallas (AMEF) con dos de las maquinarias o equipo que se obtuvieron con más problemáticas. 
Actividad 6: Análisis de los resultados: Se hizo una comparación del diagnóstico inicial con los resultados finales, y se obtuvo una gran diferencia favorable ya que disminuyeron los mantenimientos correctivos, disminuyendo los paros por mantenimientos correctivos y desarrollando un compromiso con el personal manteniendo las áreas ordenadas, clasificadas y limpias.

\section{Resultados}

\section{Análisis y recolección de datos}

Se hizo un levantamiento y documentación de la maquinaria, documentando el código, marca, modelo e imagen de cada uno de los equipos, de la planta de mezclado y distribución, dando un total de 20 equipos de análisis. En la tabla 1, se muestra un ejemplo de esta información.

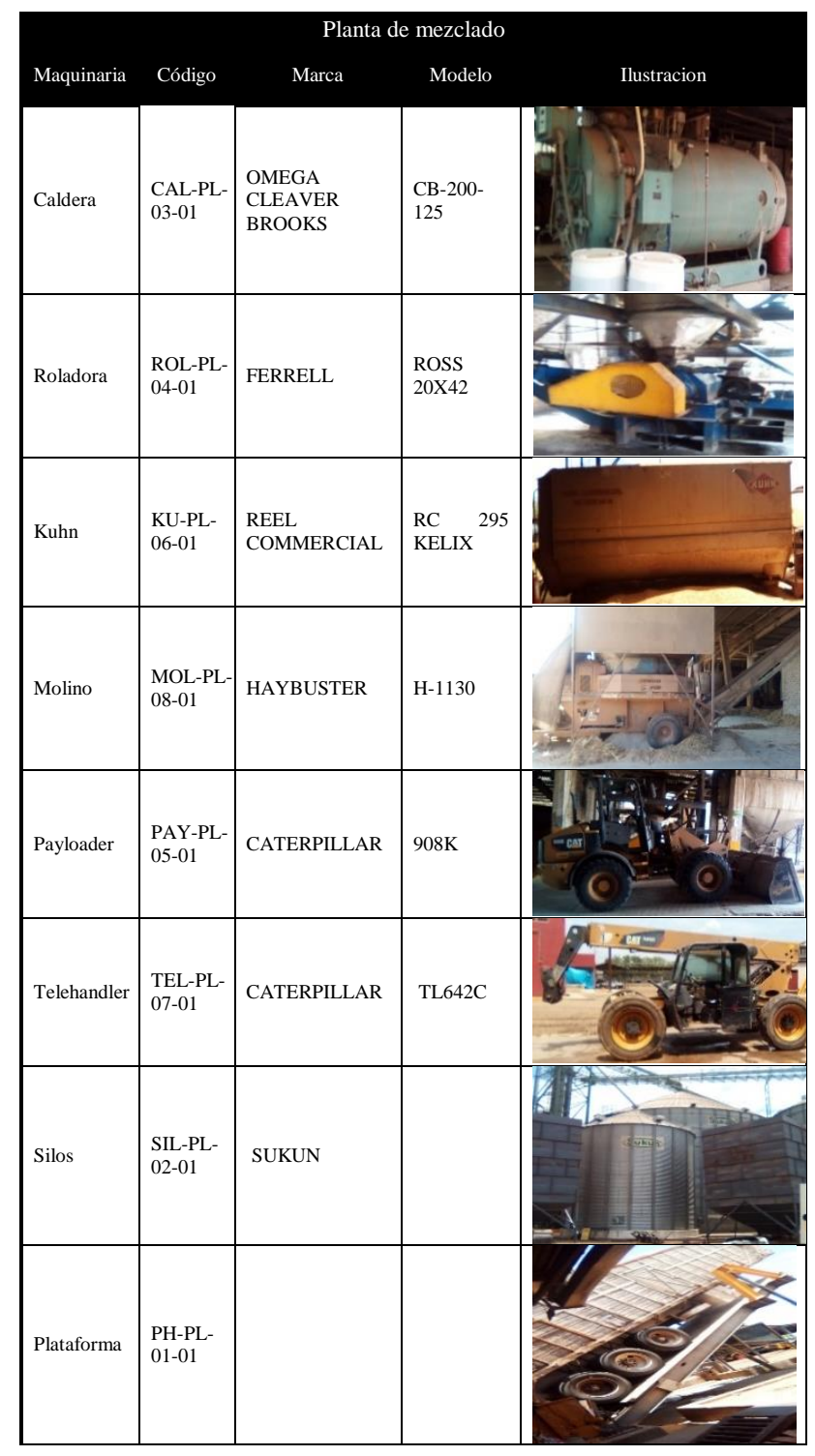

Tabla 1 Maquinaria de planta de alimento Fuente: Parra, 2018

\section{Implementación de las 5's \\ Implementación de la metodología 5's en el taller y planta}

Para la implementación de esta metodología fue necesario iniciar por dar un recorrido por el taller de mantenimiento y planta de alimentos. Como primera actividad se optó por la limpieza del taller tirando basura y desechos que ya no servían y solo hacían estorbo a los trabajadores. Posteriormente se clasificaron garrafones de aceites, mesas de trabajo y los cajones de herramientas por cada trabajador, como el del soldador, el mecánico y eléctrico. También se ordenaron las herramientas de cada trabajador para que a estos se les facilite más el encontrar las herramientas, como algunas llaves ordenadas por numeración. En conjunto con la organización y orden de herramientas y cajones en el taller, se colocó señalización para identificar las distintas cosas como cajones de herramientas con sus respectivos nombre y colores para identificarlos fácilmente. Por último se llevó a cabo una plática para concientizar a los trabajadores de mantener el orden, limpieza y clasificación de sus propias cosas y área de trabajo, creando una buena cultura y respeto entre todos los compañeros de trabajo. (Ver figuras $1 \mathrm{y} 2$ ).

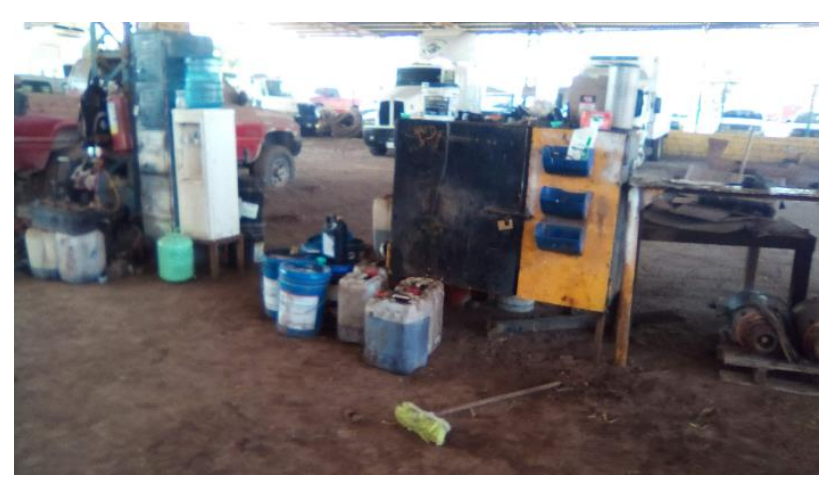

Figura 1 Área de taller antes de la implementación Fuente: Parra, 2018

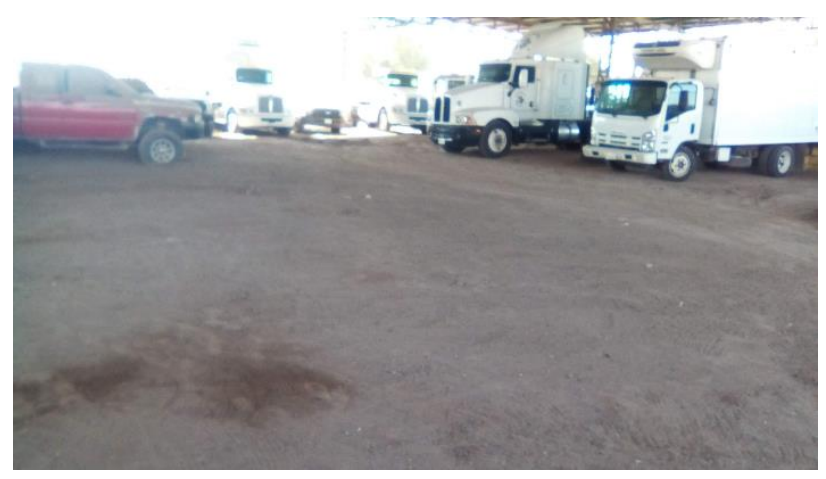

Figura 2 Área de taller después de la implementación Fuente: Parra, 2018 


\section{Implementación de la metodología 5’s en bodega y almacén}

Se inició por recabar tuercas, tornillos, huasas y empaques que estabas dispersos por el suelo. Posteriormente se clasificaron aretes para ganado por colores y se clasificaron en una sola columna en estantes. (Ver figura 3 y 4 ).

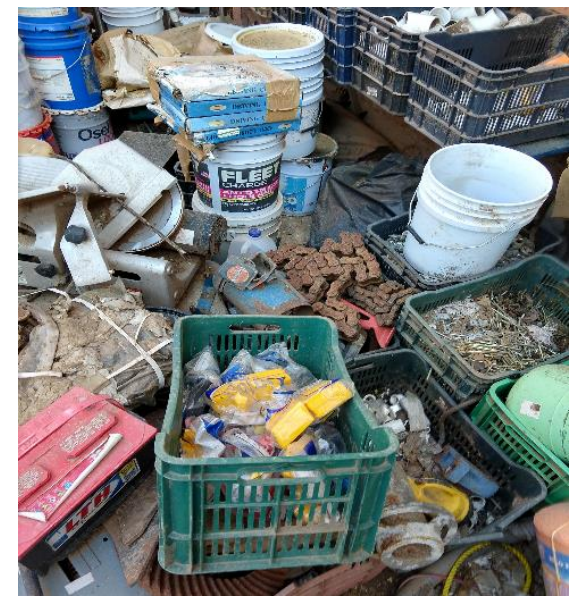

Figura 3 Área de bodega antes de la implementación Fuente: Parra, 2018
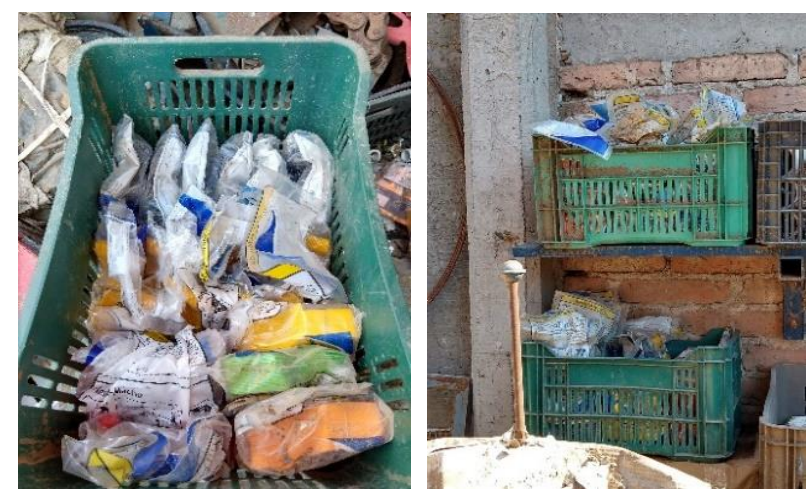

Figura 4 Área de bodega después de la implementación Fuente: Parra, 2018

Se clasificaron piezas de plomería por numeración de medida como tubos y codos de PVC. Se acomodaron y se clasificaron bebederos que estaban en diferentes partes al igual que algunas llantas. Se identificaron cubetas de vitaminas para el alimento del ganado, así como cubetas de aceites, grasas, lubricantes y pinturas. También se colocaron mecates, cables y mangueras en un lugar específico.

\section{Investigación del mantenimiento de los equipos}

Se realizó una investigación de las fichas técnicas de la maquinaria y equipos utilizados en planta de alimento con la finalidad de identificar los tipos de refacciones y tiempos de sustitución preventiva.
Se muestra un ejemplo de la ficha técnica de un Payloader Caterpillar 908k en la figura 5

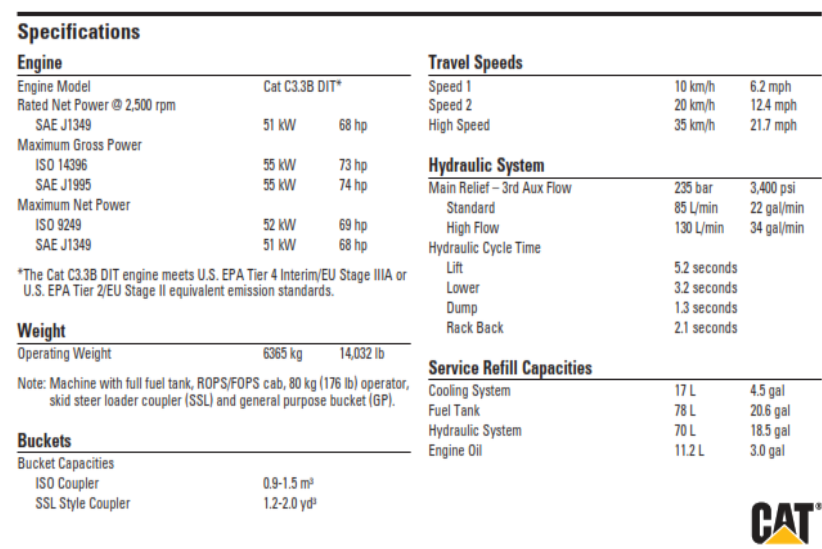

Figura 5 Ficha técnica Payloader Caterpillar 908k Fuente: Ancajima, 2004

\section{Diseño del plan de mantenimiento total de producción}

Se diseñaron para cada uno de los equipos una hoja en Excel, con las actividades, diarias, semanales y mensuales de acuerdo a la investigación previa de los requerimiento técnicos de los equipos y su buen funcionamiento. Se muestra el ejemplo de la volcadora hidráulica en la tabla 2.

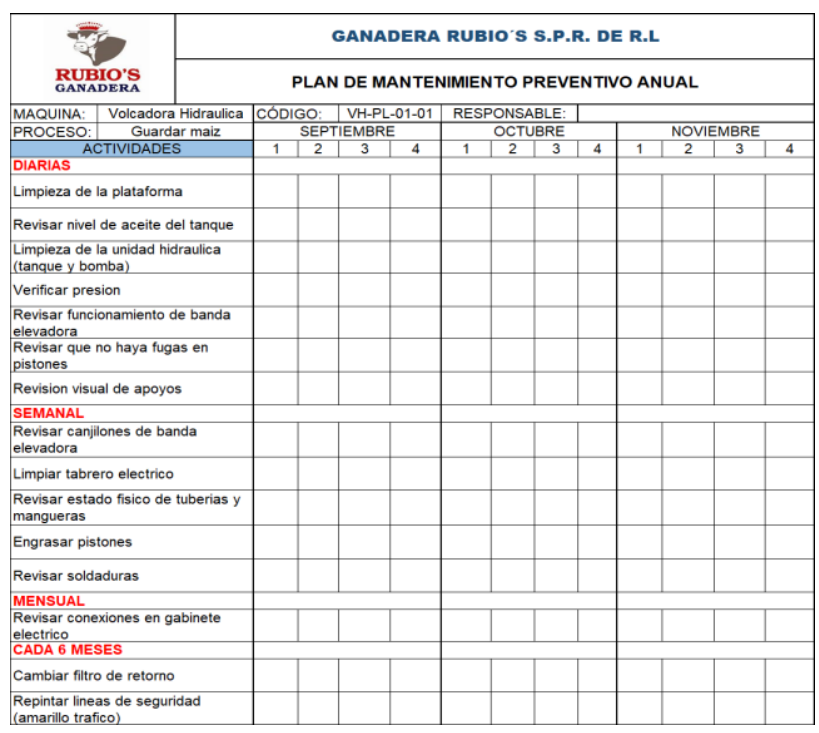

Tabla 2 Propuesta de plan anual de mantenimiento (hoja para volcadora hidráulica

Fuente: Parra, 2018

Además de diseño una lista de cotejo, denominada bitácora diaria de mantenimiento preventivo, donde se marca con una $\checkmark$ si se lleva a cabo la actividad, de lo contrario marcar con una $\mathrm{X}$, con la finalidad de verificar si se están llevando a cabo los mantenimiento programados. Se muestra un ejemplo para la volcadora hidráulica en la tabla 3.

HUMARÁN-SARMIENTO, Viridiana, PARRA-TELLEZ, Fredd Eduardo y CASTRO-LEAL, Wilfrido. Diseño e implementación de un plan de mantenimiento, basado en la metodología TPM, en planta productora de alimento balanceado para ganado bovino. Revista de Ingeniería Industrial. 2019 


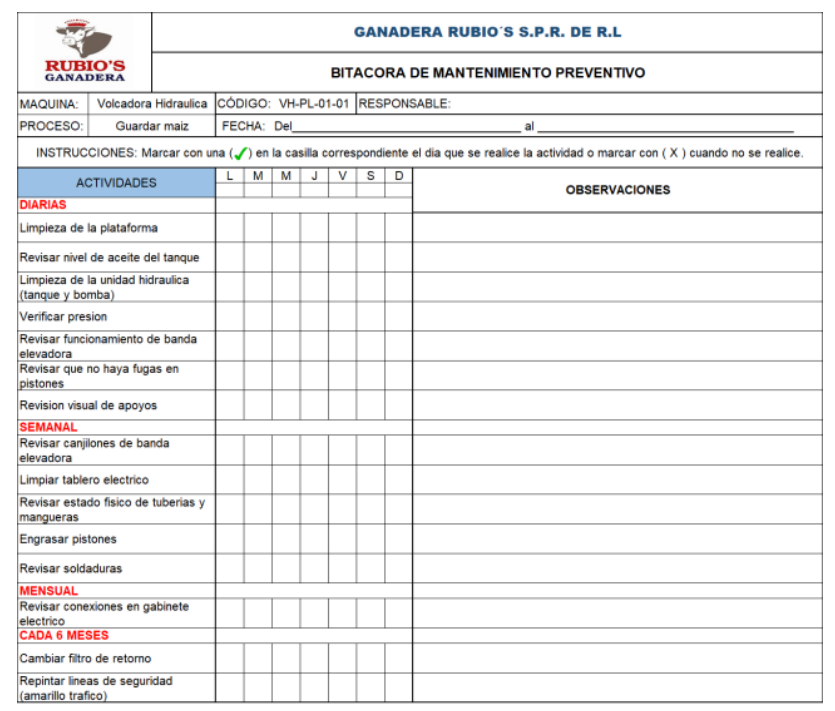

OBSERVACIONES GENERALES

\section{Nombre y firma del encargado de planta}

Tabla 3 Propuesta de bitácora de mantenimiento (hoja para volcadora hidráulica

Fuente: Parra, 2018

\section{Implementación del plan de mantenimiento total de producción}

Durante los meses de marzo y abril del 2019, se llevó a cabo la implementación del plan de mantenimiento preventivo de los 20 equipos bajo estudio, marcando con gris las celdas correspondientes a las actividades programadas y realizadas. Se muestran 3 ejemplos en las tablas 4, 5 y 6 .

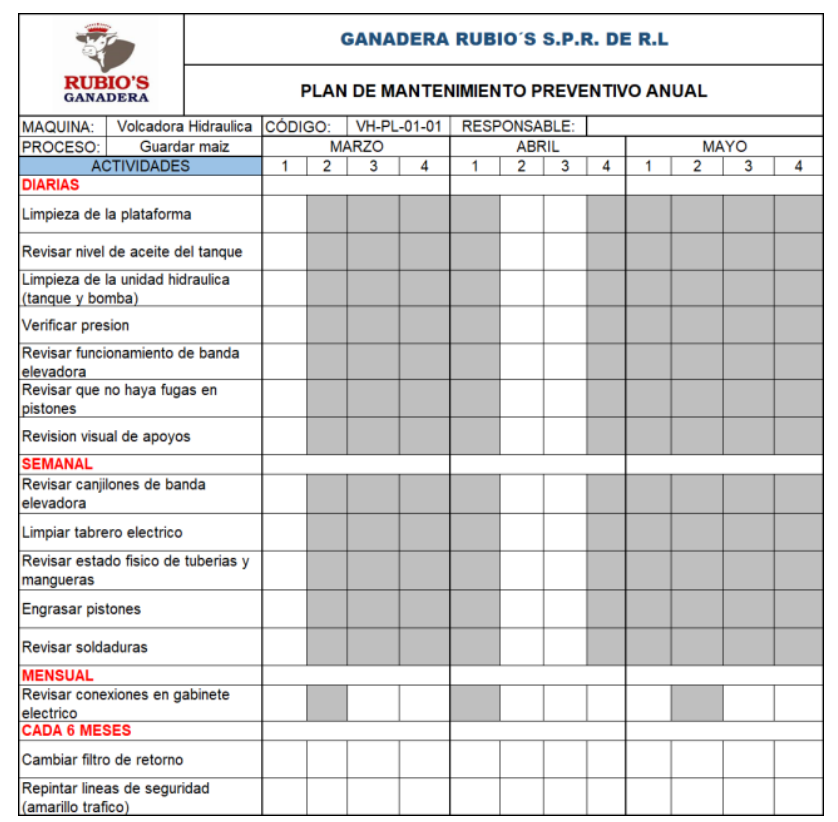

Tabla 4 Plan de Mantenimiento preventivo anual Volcadora hidráulica Fuente: Parra, 2018

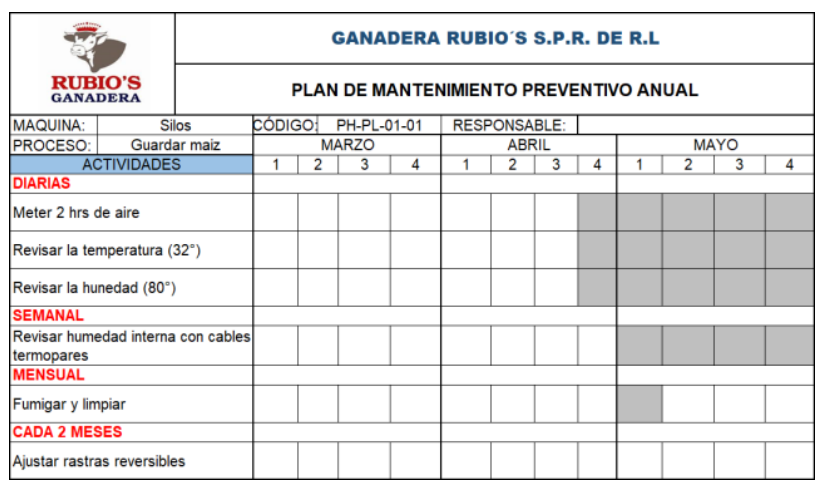

Tabla 5 Plan de Mantenimiento preventivo anual Silos Fuente: Parra, 2018

\begin{tabular}{|l|l|l|l|l|l|l|l|l|l|l|l|}
\hline & \multicolumn{7}{|c|}{ GANADERA RUBIO'S S.P.R. DE R.L } \\
\hline
\end{tabular}

Tabla 6 Plan de Mantenimiento preventivo anual Caldera Fuente: Parra, 2018

En la tabla 7 se muestran los mantenimientos correctivos que se produjeron durante los recorridos de los dos meses y con ello las correcciones realizadas y algunas que no se llegaron a realizarse.

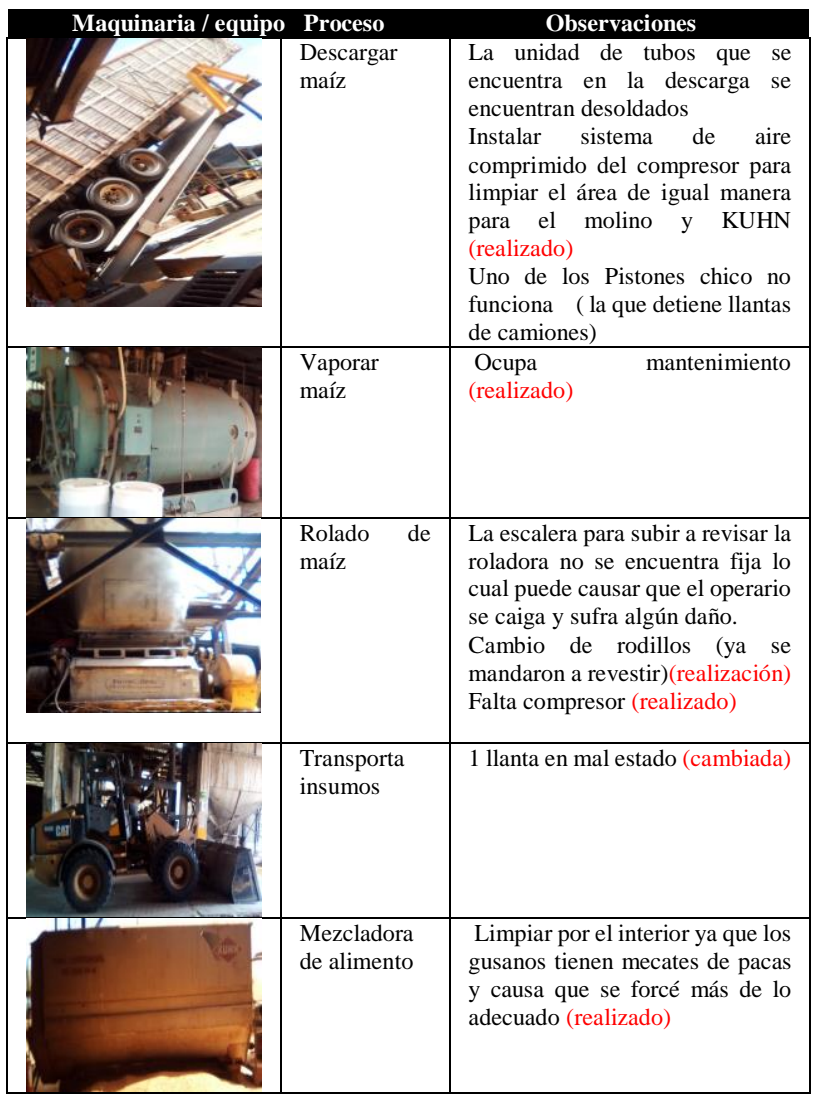

HUMARÁN-SARMIENTO, Viridiana, PARRA-TELLEZ, Freddy Eduardo y CASTRO-LEAL, Wilfrido. Diseño e implementación de un plan de mantenimiento, basado en la metodología TPM, en planta productora de alimento balanceado para ganado bovino. Revista de Ingeniería Industrial. 2019 


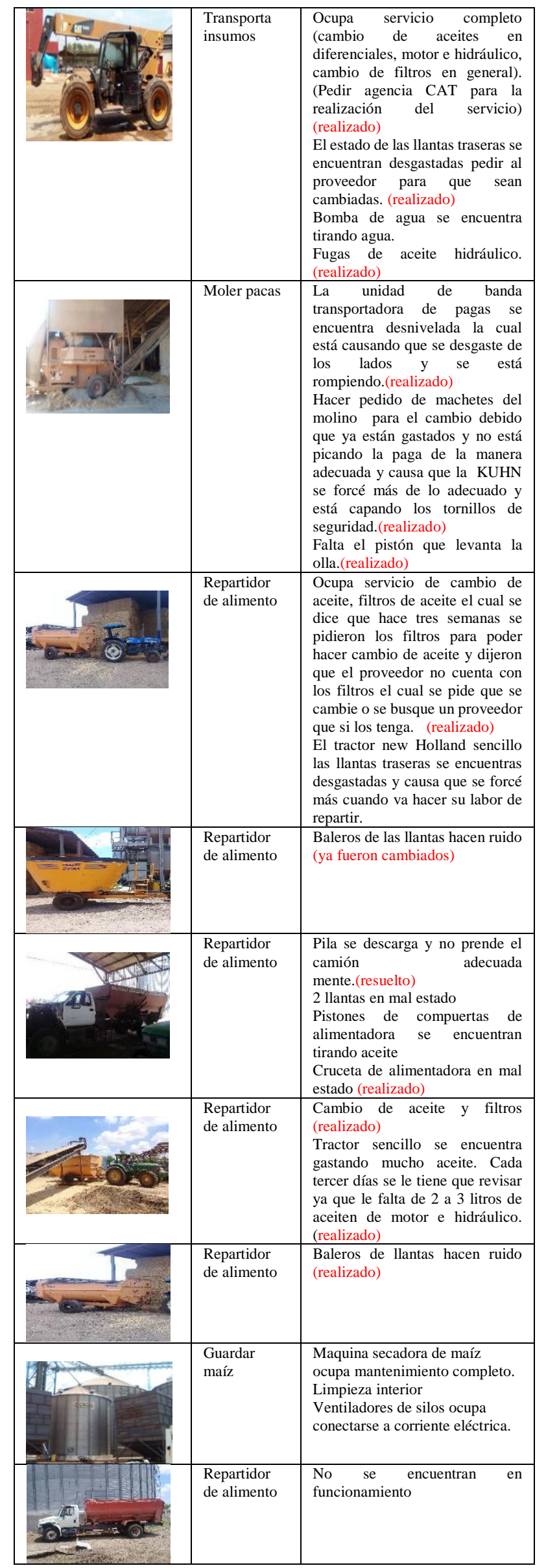

Tabla 7 Mantenimientos correctivos Fuente: Castro, 2019
Mediante la observación de las problemáticas que se obtuvieron durante estos dos meses se realizó un Análisis de Modo y Efecto de las fallas (AMEF), siguiendo la metodología de (Gutiérrez, 2009) con dos de las maquinarias o equipo que se obtuvieron con más problemáticas como lo es el camión international repartidor y molino de paja.

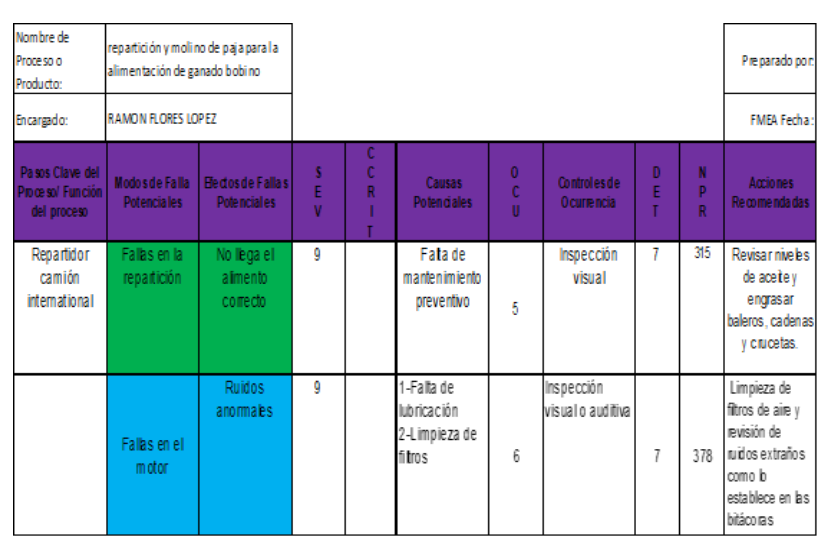

Tabla 8 Resultados de (AMEF-1)

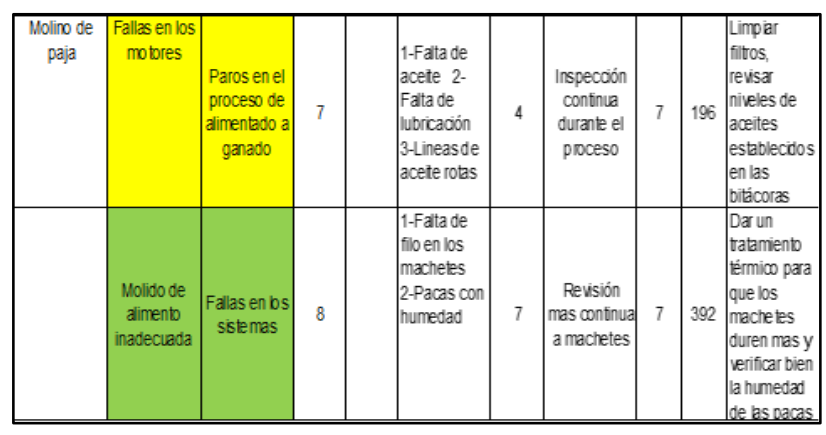

Tabla 9 Resultados de (AMEF-2)

Como se puede observar en Análisis de Modo y Efecto de las fallas (AMEF) las causas principales es que los operarios no les dan el mantenimiento preventivo adecuado a su maquinaria o equipo que les corresponden, como lo es la limpieza de los filtros o engrasado de baleros o cadenas como lo establecen en las bitácoras correspondiente a cada uno de ellos. Por otra parte como recomendación de Análisis de Modo y Efecto de las fallas (AMEF) se recomienda que al molino de paja a los machetes que estos llevan para el molido se programe un tratamiento térmico para que aumentar su dureza, y no se estén volteando o cambiando rápidamente y también verificar el parámetro de calidad de humedad de las pacas, para que el corte de los machetes no tengan desgaste prematuro. De igual manera si el molino de paja se encuentra con una fuga de aceite reparar rápidamente para que no se encuentre derramando mucho líquido hidráulico y cause que se averíe otras partes del molino a causa de falta de aceite. 
Por otra parte, una de las medidas de prevención para que disminuyan los mantenimientos correctivos, fue la entrega de las bitácoras al responsable de planta para la asignación de responsabilidades de equipo y maquinaria a cada trabajador que cuenta en dicha área para que fuesen realizadas por cada uno de ellos.

\section{Anexos}

Se realizó un informe en la planta de alimento respecto a los extintores que se encuentran en la planta de alimento y en el área de mantenimiento y se entregó dicho informe a los responsables de dichas áreas.

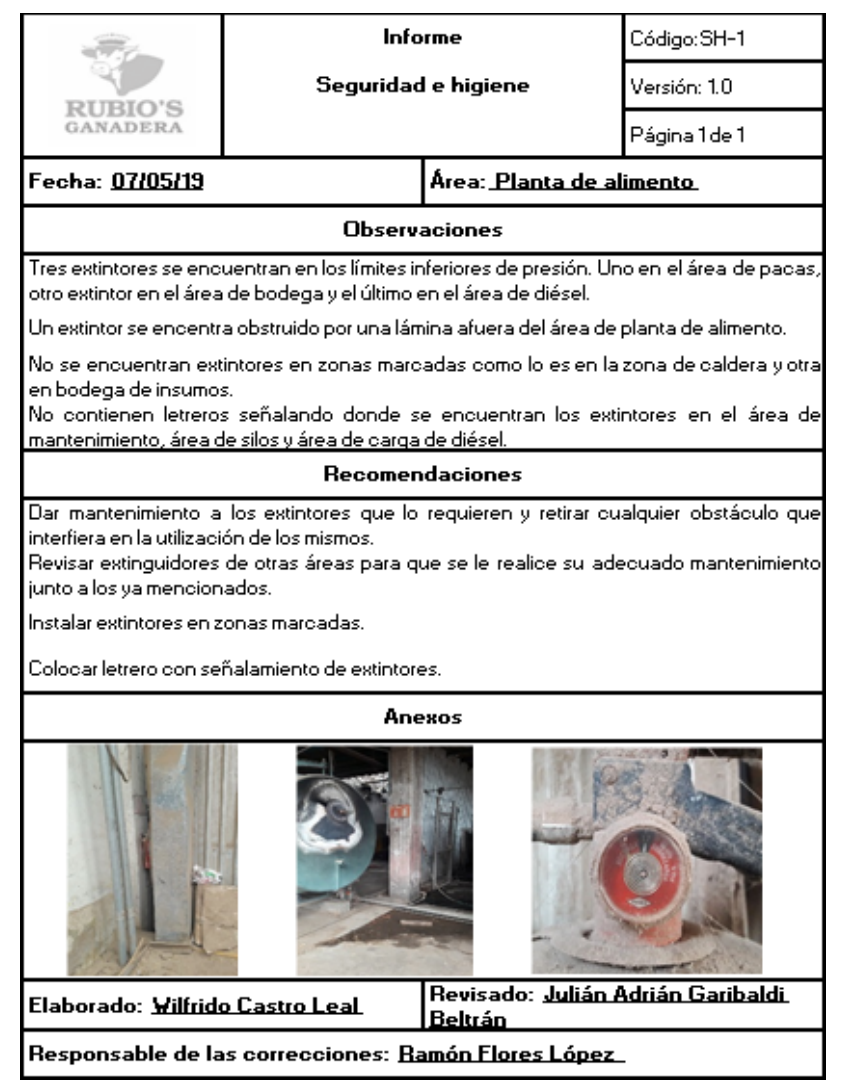

Tabla 10 Informe de seguridad e higiene

\section{Agradecimientos}

Al Tecnológico Nacional de México por el apoyo financiero otorgado para la realización de este proyecto, a través de la autorización de la convocatoria 2018-2 “Apoyo a la investigación científica y tecnológica en los programas educativos de los institutos tecnológicos federales, descentralizados y centros" vinculado con el Instituto Tecnológico Superior de Guasave (ITSG) y la empresa Ganadera Rubio’S.P. de R.L de C.V.
Reconocimiento al equipo de trabajo que formo parte de esta investigación, docentes, alumnos, personal operativo del ITSG y de la empresa beneficiada.

\section{Conclusiones}

TPM no es solo una herramienta de Ingeniería para mejorar los sistemas de mantenimiento en una empresa, porque la base para que sea exitosa la implementación de Mantenimiento Productivo Total en cualquier organización es el factor humano ya que de este depende el éxito o fracaso del proceso. Por lo tanto antes de que en las empresas se pretenda aplicar esta cultura, se debe preparar al personal lo suficiente y empoderarlo del tema para que este se motive y se entusiasme con los beneficios que les va a aportar este cambio.

Toda la organización debe entender que TPM es una implementación a largo plazo y que es un proceso de mejoramiento continuo, es decir que siempre se está mejorando, en este caso cero averías, cero defectos, cero daños. Así como el plan de mantenimiento preventivo elaborado se verán cambios a largo o mediano plazo.

\section{Sugerencias}

- Seguir la bitácora de mantenimiento preventivo semanal para disminuir o reducir el mantenimiento correctivo diariamente en las maquinas.

- $\quad$ Estar al pendiente de la programación del Plan de Mantenimiento Preventivo Anual para realizar los mantenimientos de acuerdo a las recomendaciones del fabricante de la máquina.

- Cambiar maquinaria antigua por maquinaria moderna, esto ayudaría a una mejor operación del operario de la máquina, mejorar la seguridad del operario, reducir averías y más disponibilidad, mayor rendimiento, mayor vida útil de las maquinas, menor consumo de energía y combustible.

- Limpieza en las áreas de trabajo constantemente.

- Considerar el cálculo de parámetros de productividad y costos, para calcular el costo-beneficio en caso de una inversión en maquinaria nueva. 


\section{Referencias}

Ancajima, V. J. (23 de Septiembre de 2004). SCRIBD. Obtenido de https://es.scribd.com/ document/80502385/252B-Minicargador

Castro, W. (2019). Implementación de un plan de mantenimiento preventivo a la maquinaria y equipo de planta de alimento para ganado bovino (Informe Técnico de Residencia Profesional de licenciatura). Instituto Tecnológico Superior de Guasave, Guasave, Sinaloa.

Guachisaca, C., \& Salazar, M. (2009). "Implementación de 5's como una metodología de mejora en una Empresa de elaboración de pinturas". ESCUELA SUPERIOR POLITECNICA DEL LITORAL, Guayaquil, Ecuador.

Gutiérrez Pulido, R. d. (2009). Control Estadistico de la calidad y seis sigma. Mexico: segunda edicion.

López, E. (2009). El Mantenimiento Productivo Total Tpm y la Importancia del Recurso Humano para su Exitosa Implementación. Bogotá.

López, J. (2017). sistemasoee. Obtenido de https://www.sistemasoee.com/oee/87-

avanzado/114-tpm

Mansilla, N. (2011). "Aplicación de la metodología de mantenimiento productivo total (tpm) para la estandarización de procesos y reducción de pérdidas en la fabricación de goma de mascar en una industria nacional". Santiago de Chile.

Marín, J., \& Martínez, R. (2012). Barreras y facilitadores de la implantación del TPM. Valencia, España.

Marrero- Hernández, R. A., Vilalta-Alonso, J.A., \& Martínez Delgado, E. (2019). Model diagnostic- maintenance planning and control. Ingeniería Industrial, 40(2),148-160.

Mendoza Mendoza, J.L. (2019). Revisión sistemática en plan de mantenimiento preventivo (Trabajo de Investigación Parcial).

Montoya, I., \& Parra, C. (2010). "Implementación del total productive management (tpm) como tecnología de gestión para el desarrollo de los procesos de maquiavicola ltda". Administracion de Negocios Internacionales Facultad de Administración Universidad del Rosario Bogota D.C., Bogota.

Parra, F. (2018). Diseño de un plan de mantenimiento, basado en la metodología TPM en la planta de alimento balanceado para ganado bovino: caso ganadera Rubio's (Tesis de licenciatura). Instituto Tecnológico Superior de Guasave, Guasave, Sinaloa.

Rojas Enciso, W.R., Pelinco, S., \& William, J. (2019). Mantenimiento autónomo para mejorar la calidad de servicio en el área de operaciones de la empresa $\mathrm{J} \& S$ Ingenieros Consultores EIRL-2018.

Sacristán, F. R. (2018). Mantenimiento total de la producción (tpm): proceso de implantación y desarrollo. Madrid, españa: fundación confemetal. 